\title{
Impacto da pandemia de SARS-CoV-2 na saúde mental de gestantes e puérperas: uma revisão integrativa
}

Impact of the SARS-CoV-2 pandemic on the mental health of pregnant and postpartum women: an integrative review

Impacto de la pandemia de SARS-CoV-2 en la salud mental de las mujeres embarazadas y posparto: una revisión integradora

Recebido: 07/08/2021 | Revisado: 12/08/2021 | Aceito: 13/08/2021 | Publicado: 16/08/2021

Martha Laura Leão dos Santos Silva

ORCID: https://orcid.org/0000-0003-2985-2461

Universidade Federal do Delta do Parnaíba, Brasil

E-mail: marthalauraleaodosssilva@gmail.com

Livia Rocha Santos

ORCID: https://orcid.org/0000-0002-2169-0835

Universidade Federal do Delta do Parnaíba, Brasil E-mail: liviarocha063@gmail.com

Bruna Maria de Carvalho Pereira ORCID: https://orcid.org/0000-0003-4527-3697 Universidade Federal do Delta do Parnaíba, Brasil E-mail: brunaluna.bc@gmail.com

Ana Vitória Meireles Veiga

ORCID: https://orcid.org/0000-0003-2247-4824

Universidade Federal do Delta do Parnaíba, Brasil E-mail: vitmei@hotmail.com

Daniela Winckler Mass

ORCID: https://orcid.org/0000-0003-3655-4040 Universidade Federal do Delta do Parnaíba, Brasil E-mail: danielamasspi@gmail.com

Marinice Saraiva Attem

ORCID: https://orcid.org/0000-0002-7460-5788 Instituto de Educação Superior Vale do Parnaíba, Brasil E-mail: mariniceattem@gmail.com

Lúcia Maria de Sousa Aguiar dos Santos ORCID: https://orcid.org/0000-0002-9316-2214 Centro Universitário UniFacid, Brasil E-mail: lmsantos@gmail.com

\begin{abstract}
Resumo
Objetivo: Analisar o impacto da pandemia na saúde mental de gestantes e puérperas relacionando os principais preditores da piora clínica psiquiátrica materna. Métodos: Revisão de Literatura Integrativa, cujos artigos foram buscados nas bases de dados Scientific Eletronic Library Online (SciELO), PUBMED e Literatura Latino-Americana e do Caribe (LILACS). Foram incluídos para a realização do estudo vinte e nove artigos publicados entre 2019 e 2021. Resultados: Com o advento da pandemia da covid-19, estudos mostram que gestantes estão vivenciando altos níveis de ansiedade e sintomas depressivos, divergindo dos tempos de pré-covid período no qual tais distúrbios psíquicos haviam menor incidência de casos relatados. Além disso, longos períodos de quarentena estão relacionados a um sofrimento psicológico mais grave. Também, foi possível observar na literatura que as mulheres que já apresentavam a saúde mental comprometida desde o início da gestação, possuem risco aumentado de desenvolver depressão pós parto, tendo, nesses casos, como fator protetor o aleitamento materno até o $3^{\circ}$ mês do puerpério. Considerações Finais: Somada a todas as mudanças e adaptações já esperadas na gravidez, as gestantes lidam com os efeitos secundários da covid-19. Assim, evidencia-se a relação direta entre a pandemia pelo SARS-CoV-2 e os sérios desafios psicológicos, que geram potencial aumento na incidência de ansiedade e depressão entre as gestantes, bem como, depressão pós parto em puérperas.
\end{abstract}

Palavras-chave: Isolamento social; Gravidez; SARS-CoV-2.

\begin{abstract}
Objective: To analyze the impact of the pandemic on the mental health of pregnant and postpartum women, listing the main predictors ofworsening maternal psychiatric clinic. Methods: Integrative Literature Review, which articles were
\end{abstract}


searched in the Scientific Electronic Library Online (SciELO), PUBMED and Latin American and Caribbean Literature (LILACS) databases. Twenty-nine published articles between 2019 and 2021 were included in this study. Results: Due to the advent of the covid-19 pandemic, studies show that pregnant women are experiencing high levels of anxiety and depressive symptoms, diverging from pre-covid period in which such psychiatric disorders had a lower incidence of reported cases. Furthermore, longer periods of quarantine are related to more severe psychological distress. Also, it was possible to observe in the literature that women who already had compromised mental health from the beginning of pregnancy are at increased risk of developing postpartum depression, in which cases breastfeeding until the 3rd month of the puerperium is a protective factor. Final Considerations: Added to all the changes and adaptations already expected in pregnancy, pregnant women deal with the side effects of covid-19. Thus, there is a direct relationship between the SARS-CoV-2 pandemic and the serious psychological challenges, which generate a potential increase in the incidence of anxiety, depression among pregnant women, as well as postpartum depression in postpartum women.

Keywords: Social isolation; Pregnancy; SARS-CoV-2.

\section{Resumen}

Objetivo: Analizar el impacto de la pandemia en la salud mental de mujeres embarazadas y posparto, enumerando los principales predictores de empeoramiento de la clínica psiquiátrica materna. Metodología: Revisión Integrativa de Literatura, cuyos artículos fueron buscados en las bases de datos de la Biblioteca Electrónica Científica en Línea (SciELO), PUBMED y Literatura Latinoamericana y del Caribe (LILACS). Se incluyeron para el estudio veintinueve artículos publicados entre 2019 y 2021. Resultados: Con el advenimiento de la pandemia covid-19, los estudios muestran que las mujeres embarazadas están experimentando altos niveles de ansiedad y síntomas depresivos, que divergen de los tiempos pre-covid. en las que tales trastornos psiquiátricos tenían una menor incidencia de casos notificados. Además, los períodos más prolongados de cuarentena están relacionados con una angustia psicológica más grave. Asimismo, se pudo observar en la literatura que las mujeres que ya tenían comprometida la salud mental desde el inicio del embarazo tienen mayor riesgo de desarrollar depresión posparto, y en estos casos la lactancia materna hasta el 3er mes del puerperio es un factor protector. Conclusión: Sumado a todos los cambios y adaptaciones que ya se esperaban en el embarazo, las mujeres embarazadas enfrentan los efectos secundarios del covid-19. Así, existe una relación directa entre la pandemia SARS-CoV-2 y los graves desafíos psicológicos, que generan un potencial aumento en la incidencia de ansiedad, depresión en mujeres embarazadas, así como depresión posparto en mujeres posparto.

Palabras clave: Aislamiento social; Embarazo; SARS-CoV-2.

\section{Introdução}

Em 2019, um novo Coronavírus surgiu e causou a pandemia de covid-19, gerando um alto número de mortes e consequências sem precedentes, dentre estas, o agravamento nos níveis de estresse, distúrbios do sono e depressão na população em geral Naurin et al. (2021). Não obstante, as mulheres grávidas e puérperas foram bastante afetadas, visto que, o período pré-natal costuma ser acompanhado de sofrimento mental materno associado a questionamentos da própria gravidez, que também envolvem o bem-estar fetal e o desfecho do parto. Além disso, um dos fatores que podem afetar a saúde mental de mulheres grávidas são inseguranças relacionadas a eventos catastróficos ou desastres naturais (Salehi, Rahimzadeh, Molaei, Zaheri, \& Esmaelzadeh-Saeieh 2020).

Nessa perspectiva, gestantes foram gravemente afetadas por epidemias anteriores. Então, alguns pesquisadores previram que a pandemia atual da covid-19 poderia ter impacto semelhante. Desta forma, com as incertezas levantadas acerca de questionamentos sobre a possibilidade de transmissão de mãe para filho, bem como o efeito de covid-19 para o feto, além da probabilidade de aumento do risco de contrair a doença ou apresentar complicações graves acarretou em impactos negativos para a saúde das gestantes e puérperas nesse período. Soma-se a isso ainda, o risco de morte e a incerteza sobre a eficácia dos tratamentos disponíveis e segurança de vacinas (Mortazavi, Mehrabad, \& KiaeeTabar 2021).

Vale destacar também que a depressão pós-parto (PPD) é um problema de saúde mental relativamente comum e grave, tendo o apoio social como um relevante fator de proteção. Outrossim, o suicídio é responsável por uma a cada cinco mortes e é a segunda principal causa de mortalidade no primeiro ano pós-parto. A gravidade dos sintomas de PPD está associada a muitos fatores biológicos e não biológicos, sendo que a doença afeta cerca de $13 \%$ a $20 \%$ das novas mães (Chrzan- 
Neste contexto, dadas as potenciais sequelas negativas de incerteza psicológica, de saúde e financeira, juntamente com o isolamento social, urge determinar a prevalência de doenças psicológicas entre grávidas durante esta pandemia e identificar fatores de proteção, para que intervenções direcionadas possam ser rapidamente implementadas (Lebel, MacKinnon, Bagshawe, Tomfohr-Madsen, \& Giesbrecht 2020).

Neste viés, o apoio social é um importante fator de proteção; porém, durante a pandemia, as novas mães são privadas de seu trabalho e de convívio social. Ademais, o contato direto prolongado com outras crianças (cuidados diários e educação em casa) e, mudança para escritório em casa podem intensificar fadiga diária e estresse. Desta forma, dificultam o equilíbrio entre vida profissional e pessoal, desencadeiam conflitos e interferem na adaptação à vida com um novo bebê. Assim, uma crise epidêmica pode limitar parte dos recursos psicológicos que constroem a saúde mental da mulher no período pós-parto (Chrzan-Dętkoś et al. 2021).

O principal objetivo deste estudo foi realizar uma síntese integrativa sobre as consequências relacionadas à covid-19, com base na análise de artigos que abordam o assunto, a fim de correlatar o impacto da pandemia e saúde mental de gestantes e puérperas. Diante do exposto, o questionamento da pesquisa urge avaliar quais os principais preditores de piora clínica da saúde mental e as situações que, eventualmente, possam condicionar a paciente ou o recém-nascido a complicações durante a gravidez e no pós-parto.

\section{Metodologia}

Trata-se de uma revisão de literatura integrativa, com o intuito de avaliar quais foram os impactos do isolamento social imposto pela pandemia do novo coronavírus na saúde mental de mulheres gestantes e puérperas.

A busca pelos artigos foi realizada nas bases de dados Scientific Eletronic Library Online (SciELO), PUBMED e Literatura Latino-Americana e do Caribe (LILACS), utilizando os descritores: "Isolamento social", "Gravidez" e "SARS-CoV2". Na pesquisa, os termos foram relacionados por meio do operador booleano AND (E).

$\mathrm{Na}$ base SciELO, ao associar os três descritores não foi encontrado nenhum resultado; em seguida, a pesquisa foi conduzida utilizando os descritores em pares. Ao associar "Isolamento social" e "Gravidez", foram encontrados 17 artigos. Com a associação "Gravidez" e "SARS-CoV-2" foram encontrados 41 artigos e associando "Isolamento social" e "SARSCoV-2" foram encontrados 53 artigos.

Ao utilizar os três descritores na base LILACS, não foram encontrados resultados. Ao associar "Isolamento social" e "Gravidez" foram encontrados seis artigos, utilizando "Gravidez" e "SARS-CoV-2" foram encontrados 64 artigos e ao associar "Isolamento social" e "SARS-CoV-2" foram encontrados 34 artigos.

Já na base de dados PUBMED, ao associar os três descritores foram encontrados 46 resultados, com "Isolamento social" e "Gravidez" foram encontrados 1.050 artigos. Ao associar "Gravidez" e "SARS-CoV-2" foram encontrados 1.944 artigos e ao pesquisar "Isolamento social" e "SARS-CoV-2" foram encontrados 1.991 artigos.

No total, foram encontrados 5.246 artigos, dentre esses, por meio da leitura do título, foram excluídos 5.164 por não abordarem diretamente o tema de estudo. Dos 82 artigos restantes, após a leitura dos resumos, foram excluídos 50 por não se enquadrarem no tema de estudo. Dessa forma, restaram 32 artigos para leitura integral, sendo eliminados três que estavam repetidos. Ao final foram incluídos 29 artigos para a realização do estudo. O fluxograma da figura 1 demonstra as etapas seguidas para a seleção de artigos.

A pesquisa ocorreu em maio de 2021 e os critérios para inclusão foram: artigos científicos nos idiomas português, inglês ou espanhol, publicados entre 2019 e 2021, disponibilizados de forma integral e que abordassem o tema de estudo. Foram excluídos materiais que não abordassem o tema de estudo ou que não fossem artigos científicos. 
Research, Society and Development, v. 10, n. 10, e484101019186, 2021

(CC BY 4.0) | ISSN 2525-3409 | DOI: http://dx.doi.org/10.33448/rsd-v10i10.19186

Figura 1 - Fluxograma detalhando cada etapa do processo de seleção de artigos para a revisão.
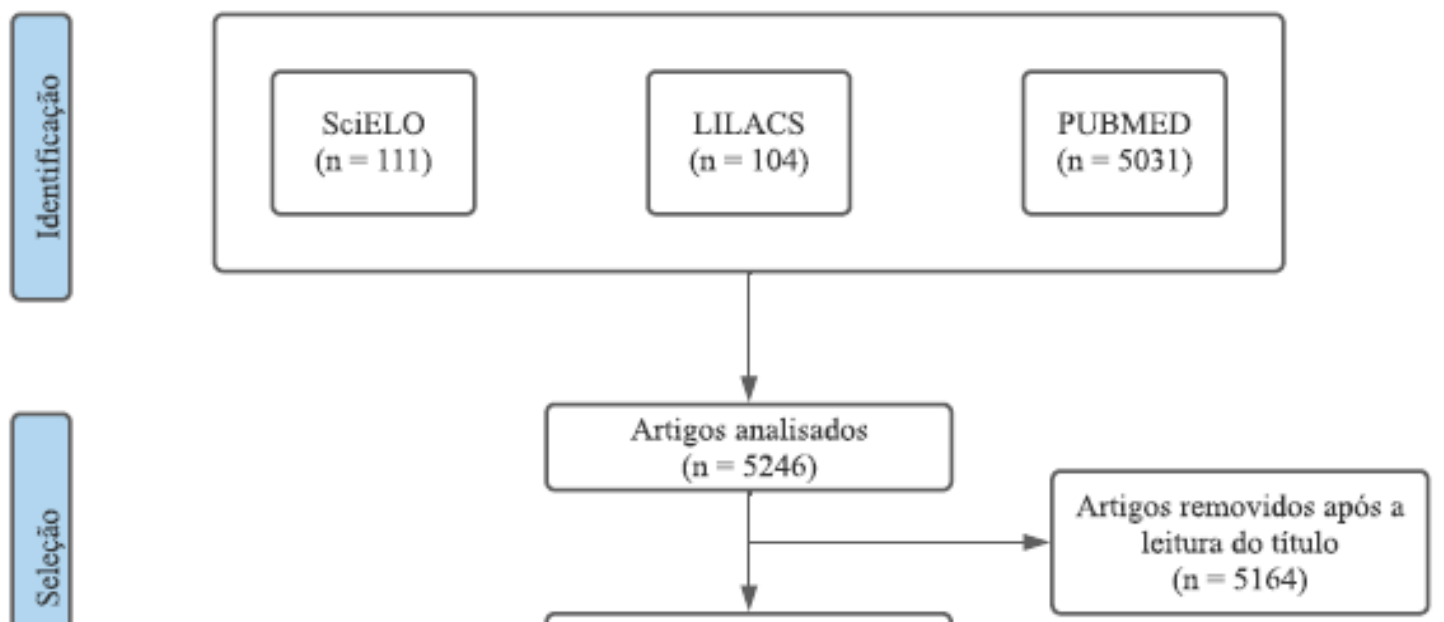

Artigos selecionados para a

leitura dos resumos $(\mathrm{n}=82)$
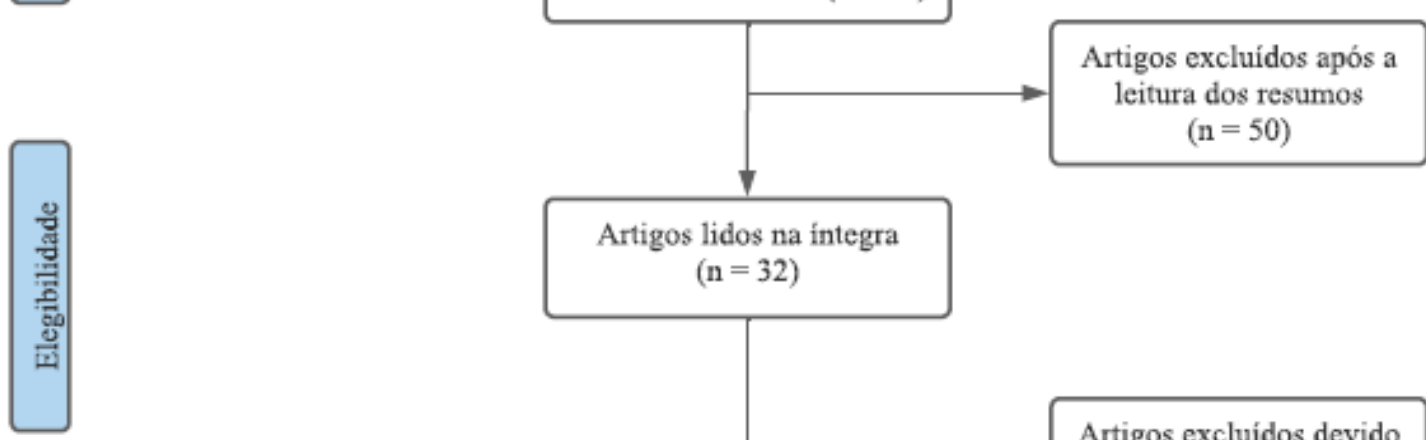

Artigos lidos na integra $(\mathrm{n}=32)$

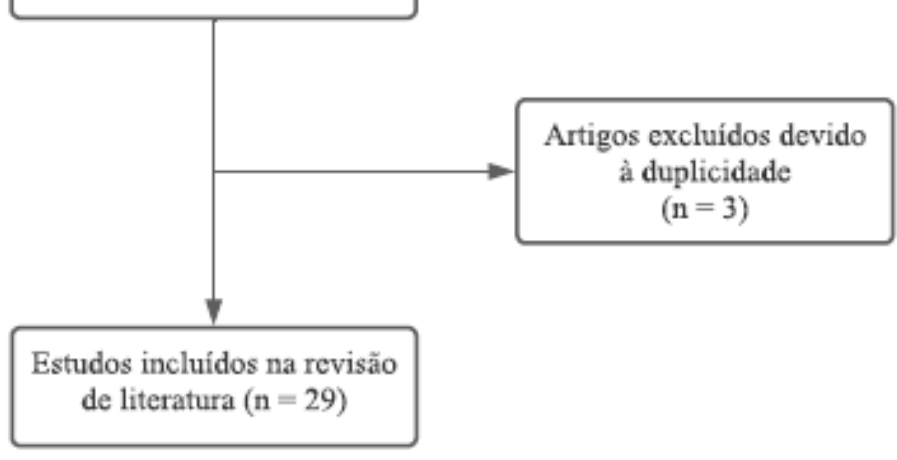

Fonte: Silva et al. (2021).

\section{Resultados e Discussão}

A amostra final da revisão foi composta por 29 artigos retirados das três bases de dados pesquisadas e que obedeciam aos critérios de inclusão. Os trabalhos utilizados estão listados com informações acerca do título e dos principais objetivos (Quadro 1). 
Quadro 1 - Artigos selecionados para a realização do estudo especificando a revista, ano de publicação e principais objetivos e resultados.

\begin{tabular}{|c|c|c|c|c|c|}
\hline Obra & TÍTULO & ANO & PERIÓDICO & OBJETIVO & ACHADOS RELEVANTES \\
\hline 1 & $\begin{array}{l}\text { The impact of the COVID- } \\
19 \text { pandemic on women's } \\
\text { mental health }\end{array}$ & 2020 & $\begin{array}{l}\text { Archives of } \\
\text { women's mental } \\
\text { health }\end{array}$ & $\begin{array}{c}\text { Revisar o que se sabe sobre o efeito } \\
\text { da pandemia na saúde mental das } \\
\text { mulheres. }\end{array}$ & $\begin{array}{l}\text { Mulheres grávidas, no pós- } \\
\text { parto, com aborto espontâneo } \\
\text { ou sofrendo violência por } \\
\text { parceiro íntimo possuem maior } \\
\text { risco de desenvolver problemas } \\
\text { de saúde mental durante a } \\
\text { pandemia. }\end{array}$ \\
\hline 2 & $\begin{array}{l}\text { Maternal mental health and } \\
\text { coping during the COVID- } \\
19 \text { lockdown in the UK: } \\
\text { Data from the COVID-19 } \\
\text { New Mum Study }\end{array}$ & 2020 & $\begin{array}{l}\text { International } \\
\text { Journal of } \\
\text { Gynecology and } \\
\text { Obstetrics }\end{array}$ & $\begin{array}{c}\text { Avaliar como as mães estão se } \\
\text { sentindo durante o confinamento e } \\
\text { identificar os caminhos potenciais } \\
\text { que podem ajudá-las. }\end{array}$ & $\begin{array}{l}\text { Para melhoria da saúde mental } \\
\text { materna, é essencial identificar } \\
\text { estratégias de prevenção para as } \\
\text { mães durante o confinamento. }\end{array}$ \\
\hline 3 & $\begin{array}{l}\text { Anxiety and post-traumatic } \\
\text { stress disorder symptoms in } \\
\text { pregnant women during the } \\
\text { covid-19 pandemic's delay } \\
\text { phase }\end{array}$ & 2020 & $\begin{array}{c}\text { Psychiatria } \\
\text { Danubina }\end{array}$ & $\begin{array}{l}\text { Determinar os sintomas de } \\
\text { ansiedade e transtorno de estresse } \\
\text { pós-traumático em mulheres } \\
\text { grávidas durante a pandemia de } \\
\text { COVID-19. }\end{array}$ & $\begin{array}{l}\text { Complicações na gravidez e a } \\
\text { situação de emprego do marido } \\
\text { foram os melhores preditores do } \\
\text { estado de ansiedade nas mães. }\end{array}$ \\
\hline 4 & $\begin{array}{l}\text { Evaluation of a remote } \\
\text { control model for pregnant } \\
\text { and post-partum patients } \\
\text { with SARS-CoV-2infection }\end{array}$ & 2020 & $\begin{array}{l}\text { Revista Chilena } \\
\text { de Obstetricia y } \\
\text { Ginecologia }\end{array}$ & $\begin{array}{l}\text { Avaliar a satisfação das pacientes } \\
\text { no seguimento remoto de grávidas e } \\
\text { puérperas com diagnóstico de } \\
\text { infecção por SARS-CoV- } 2 \text {. }\end{array}$ & $\begin{array}{l}\text { No contexto da pandemia de } \\
\text { SARS-CoV-2, o } \\
\text { acompanhamento remoto de } \\
\text { pacientes grávidas e puérperas } \\
\text { foi avaliado positivamente. }\end{array}$ \\
\hline 5 & $\begin{array}{l}\text { Elevated depression and } \\
\text { anxiety symptoms among } \\
\text { pregnant individuals during } \\
\text { the COVID-19 pandemic }\end{array}$ & 2020 & $\begin{array}{l}\text { Journal of } \\
\text { Affective } \\
\text { Disorders }\end{array}$ & $\begin{array}{l}\text { Avaliar sintomas de ansiedade e } \\
\text { depressão em gestantes durante a } \\
\text { pandemia de COVID- } 19 .\end{array}$ & $\begin{array}{c}\text { Foram encontrados sintomas de } \\
\text { ansiedade e depressão elevados } \\
\text { nas gestantes em comparação } \\
\text { com coortes de gravidez pré- } \\
\text { pandêmicas semelhantes. }\end{array}$ \\
\hline 6 & $\begin{array}{l}\text { COVID-19-related anxiety } \\
\text { and concerns expressed by } \\
\text { pregnant and postpartum } \\
\text { women-a survey among } \\
\text { obstetricians }\end{array}$ & 2020 & $\begin{array}{c}\text { Archives of } \\
\text { Women's Mental } \\
\text { Health }\end{array}$ & $\begin{array}{c}\text { Descrever as ansiedades que } \\
\text { mulheres grávidas relataram aos } \\
\text { obstetras durante a pandemia de } \\
\text { COVID-19. }\end{array}$ & $\begin{array}{c}\text { Obstetras sentiram a } \\
\text { necessidade de recursos como } \\
\text { vídeos para lidar com a } \\
\text { ansiedade entre mulheres no } \\
\text { período perinatal durante a } \\
\text { pandemia. }\end{array}$ \\
\hline 7 & $\begin{array}{l}\text { Amamentação durante a } \\
\text { Pandemia COVID-19: } \\
\text { Revisão Narrativa }\end{array}$ & 2020 & $\begin{array}{l}\text { Ecuadorian } \\
\text { Journal of } \\
\text { Pediatrics }\end{array}$ & $\begin{array}{c}\text { Delinear o papel do aleitamento } \\
\text { materno em mulheres com COVID- } \\
\text { 19, suspeita ou confirmação } \\
\text { diagnóstica. }\end{array}$ & $\begin{array}{l}\text { As recomendações vigentes } \\
\text { encorajam a amamentação para } \\
\text { lactantes com suspeita ou } \\
\text { confirmação de covid-19 devido } \\
\text { aos inúmeros benefícios deste } \\
\text { ato, porém as medidas de } \\
\text { biossegurança devem ser } \\
\text { seguidas corretamente. }\end{array}$ \\
\hline 8 & $\begin{array}{c}\text { Psychometric properties of } \\
\text { the Pandemic-Related } \\
\text { Pregnancy Stress Scale } \\
\text { (PREPS) }\end{array}$ & 2020 & $\begin{array}{c}\text { Journal of } \\
\text { Psychosomatic } \\
\text { Obstetrics and } \\
\text { Gynecology }\end{array}$ & $\begin{array}{c}\text { Testar as propriedades } \\
\text { psicométricas de um instrumento } \\
\text { para avaliar o estresse relacionado a } \\
\text { pandemia de COVID-19 em } \\
\text { mulheres grávidas. }\end{array}$ & $\begin{array}{l}\text { O PREPS é um instrumento útil } \\
\text { na avaliação do estresse pré- } \\
\text { natal durante a pandemia e de } \\
\text { como essa situação pode afetar } \\
\text { as mulheres e seus filhos. }\end{array}$ \\
\hline 9 & $\begin{array}{l}\text { A multidisciplinary } \\
\text { telemedicine model for } \\
\text { management }\end{array}$ & 2020 & $\begin{array}{l}\text { The American } \\
\text { Journal of } \\
\text { Obstetrics \& } \\
\text { Gynecology }\end{array}$ & $\begin{array}{c}\text { Descrever a viabilidade e desfechos } \\
\text { de processos associados a uma } \\
\text { telemedicina multidisciplinar para } \\
\text { gestão de pacientes obstétricos com } \\
\text { conhecida exposição e sintomas de } \\
\text { COVID- } 19 \text {. }\end{array}$ & $\begin{array}{l}\text { Um modelo de vigilância por } \\
\text { telemedicina para gestantes com } \\
\text { sintomas de covid-19 previne } \\
\text { exposições evitáveis da equipe } \\
\text { de saúde e da paciente. }\end{array}$ \\
\hline
\end{tabular}




\begin{tabular}{|c|c|c|c|c|c|}
\hline 10 & $\begin{array}{l}\text { The relationship among } \\
\text { fear and anxiety of } \\
\text { COVID-19, pregnancy } \\
\text { experience, and mental } \\
\text { health disorder in pregnant } \\
\text { women: A structural } \\
\text { equation model }\end{array}$ & 2020 & $\begin{array}{l}\text { Brain and } \\
\text { Behavior }\end{array}$ & $\begin{array}{l}\text { Analisar o medo e a ansiedade } \\
\text { causados pelo coronavírus, a } \\
\text { experiência da gravidez e a saúde } \\
\text { mental de mulheres grávidas. }\end{array}$ & $\begin{array}{l}\text { O medo da covid-19 tem um } \\
\text { efeito direto sobre a ansiedade e } \\
\text { está relacionado a transtornos } \\
\text { mentais durante a gravidez. }\end{array}$ \\
\hline 11 & $\begin{array}{l}\text { Atendimento de gestantes } \\
\text { na atenção primária à saúde } \\
\text { pela enfermagem durante a } \\
\text { pandemia do SARS-COV-2 }\end{array}$ & 2020 & Nursing & $\begin{array}{l}\text { Descrever o papel do enfermeiro na } \\
\text { realização de consultas pré-natal } \\
\text { durante a pandemia no âmbito da } \\
\text { Atenção Primária à Saúde. }\end{array}$ & $\begin{array}{l}\text { Os enfermeiros possuem papel } \\
\text { fundamental na orientação da } \\
\text { população em geral, em } \\
\text { especial de gestantes. Este papel } \\
\text { facilita a intervenção contínua } \\
\text { do profissional de saúde durante } \\
\text { o pré-natal. }\end{array}$ \\
\hline 12 & $\begin{array}{l}\text { Does having a high-risk } \\
\text { pregnancy influence } \\
\text { anxiety level during the } \\
\text { COVID-19 pandemic? }\end{array}$ & 2020 & $\begin{array}{c}\text { European Journal } \\
\text { of Obstetrics and } \\
\text { Gynecology and } \\
\text { Reproductive } \\
\text { Biology }\end{array}$ & $\begin{array}{l}\text { Analisar o nível de mudança de } \\
\text { ansiedade durante a pandemia de } \\
\text { COVID-19 em mulheres grávidas } \\
\text { com e sem indicadores de alto risco. }\end{array}$ & $\begin{array}{l}\text { A prevalência de ansiedade } \\
\text { durante a pandemia é maior na } \\
\text { gravidez de alto risco em } \\
\text { comparação com gestantes sem } \\
\text { fatores de risco. }\end{array}$ \\
\hline 13 & $\begin{array}{l}\text { Distress and anxiety } \\
\text { associated with COVID-19 } \\
\text { among Jewish and Arab } \\
\text { pregnant women in Israel }\end{array}$ & 2020 & $\begin{array}{c}\text { Journal of } \\
\text { Reproductive and } \\
\text { Infant Psychology }\end{array}$ & $\begin{array}{c}\text { Explorar o sofrimento psicológico e } \\
\text { a ansiedade relacionada a COVID- } \\
19 \text { em mulheres grávidas durante a } \\
\text { crise. }\end{array}$ & $\begin{array}{l}\text { Os níveis de ansiedade em } \\
\text { gestantes durante a pandemia } \\
\text { são bastante elevados e se } \\
\text { relacionam com vários fatores, } \\
\text { dentre eles estão o medo de } \\
\text { infecção de membros da } \\
\text { família, medo da própria } \\
\text { infecção e a saúde do feto. }\end{array}$ \\
\hline 14 & $\begin{array}{l}\text { Protocolo de atenção ao } \\
\text { parto, puerpério e aborto } \\
\text { durante a pandemia de } \\
\text { COVID-19 }\end{array}$ & 2020 & $\begin{array}{l}\text { Revista Brasileira } \\
\text { de Ginecologia e } \\
\text { Obstetrícia }\end{array}$ & $\begin{array}{l}\text { Fornecer apoio técnico e científico } \\
\text { aos obstetras brasileiros com } \\
\text { relação aos cuidados no parto, pós- } \\
\text { parto e aborto durante a pandemia. }\end{array}$ & $\begin{array}{l}\text { Fosfato de oseltamivir é } \\
\text { amplamente recomendado nos } \\
\text { estágios iniciais de gripe em } \\
\text { gestantes. }\end{array}$ \\
\hline 15 & $\begin{array}{l}\text { Social distancing } \\
\text { compliance under covid-19 } \\
\text { pandemic and mental } \\
\text { health impacts: A } \\
\text { population-based study }\end{array}$ & 2020 & $\begin{array}{l}\text { International } \\
\text { Journal of } \\
\text { Environmental } \\
\text { Research and } \\
\text { Public Health }\end{array}$ & $\begin{array}{c}\text { Analisar a associação do } \\
\text { distanciamento social com a saúde } \\
\text { mental durante a pandemia de } \\
\text { COVID-19. }\end{array}$ & $\begin{array}{c}\text { A população idosa e com menor } \\
\text { escolaridade pode ser mais } \\
\text { vulnerável aos sintomas } \\
\text { depressivos decorrentes das } \\
\text { mudanças bruscas nas regras } \\
\text { sociais. }\end{array}$ \\
\hline 16 & $\begin{array}{c}\text { The influence of } \\
\text { psychological response and } \\
\text { security sense on } \\
\text { pregnancy stress during the } \\
\text { outbreak of coronavirus } \\
\text { disease 2019: A mediating } \\
\text { model }\end{array}$ & 2020 & $\begin{array}{c}\text { Journal of Clinical } \\
\text { Nursing }\end{array}$ & $\begin{array}{l}\text { Observar o estado psicológico de } \\
\text { mulheres grávidas durante a } \\
\text { pandemia de COVID-19 e testar um } \\
\text { modelo hipotético que estima a } \\
\text { influência da resposta psicológica a } \\
\text { COVID-19. }\end{array}$ & $\begin{array}{l}\text { Medo e depressão foram as } \\
\text { respostas psicológicas mais } \\
\text { comuns entre as mulheres } \\
\text { grávidas durante a pandemia de } \\
\text { covid-19. Outrossim, a equipe } \\
\text { de saúde pode auxiliar na } \\
\text { redução do estresse em } \\
\text { mulheres grávidas. }\end{array}$ \\
\hline 17 & $\begin{array}{l}\text { A survey of postpartum } \\
\text { depression and health care } \\
\text { needs among Chinese } \\
\text { postpartum women during } \\
\text { the pandemic of COVID-19 }\end{array}$ & 2021 & $\begin{array}{l}\text { Archives of } \\
\text { Psychiatric } \\
\text { Nursing }\end{array}$ & $\begin{array}{l}\text { Investigar os fatores de risco da } \\
\text { depressão pós-parto e as } \\
\text { necessidades de saúde entre as } \\
\text { mulheres chinesas no puerpério } \\
\text { durante a pandemia de COVID-19. }\end{array}$ & $\begin{array}{l}\text { Mulheres na faixa etária de } 25 \text { a } \\
34 \text { anos, com histórico de } \\
\text { aborto e altos níveis de estresse } \\
\text { possuem maior risco de } \\
\text { depressão pós-parto. }\end{array}$ \\
\hline 18 & $\begin{array}{l}\text { Development and } \\
\text { validation of a } \\
\text { questionnaire to assess } \\
\text { socio-behavioural impact } \\
\text { of COVID-19 on the } \\
\text { general population }\end{array}$ & 2021 & $\begin{array}{l}\text { Diabetes and } \\
\text { Metabolic } \\
\text { Syndrome: } \\
\text { Clinical Research } \\
\text { and Reviews }\end{array}$ & $\begin{array}{c}\text { Desenvolver uma ferramenta válida } \\
\text { e confiável para avaliar as } \\
\text { mudanças sociocomportamentais } \\
\text { devido a COVID na população em } \\
\text { geral. }\end{array}$ & $\begin{array}{c}\text { Ferramentas para avaliar } \\
\text { mudanças comportamentais } \\
\text { durante a pandemia são } \\
\text { importantes para o } \\
\text { desenvolvimento de estratégias } \\
\text { eficazes. }\end{array}$ \\
\hline
\end{tabular}




\begin{tabular}{|c|c|c|c|c|c|}
\hline 19 & $\begin{array}{l}\text { Experiences of } \\
\text { breastfeeding during } \\
\text { COVID-19: Lessons for } \\
\text { future practical and } \\
\text { emotional support }\end{array}$ & 2021 & $\begin{array}{l}\text { Maternal and } \\
\text { Child Nutrition }\end{array}$ & $\begin{array}{l}\text { Entender o impacto da pandemia } \\
\text { sobre a capacidade de amamentar. }\end{array}$ & $\begin{array}{l}\text { Mães com menor escolaridade, } \\
\text { menor renda e de etnia negra } \\
\text { são mais propensas a } \\
\text { interromperem a amamentação } \\
\text { em decorrência da pandemia. }\end{array}$ \\
\hline 20 & $\begin{array}{l}\text { Experiences of pregnant } \\
\text { mothers using a social } \\
\text { media based antenatal } \\
\text { support service during the } \\
\text { COVID-19 lockdown in the } \\
\text { UK: Findings from a user } \\
\text { survey }\end{array}$ & 2021 & BMJ Open & $\begin{array}{c}\text { Explorar as experiências de mães } \\
\text { que estavam usando uma mídia } \\
\text { social existente no Serviço Nacional } \\
\text { de Saúde baseado no serviço de } \\
\text { suporte pré-natal durante as fases } \\
\text { iniciais do confinamento no Reino } \\
\text { Unido. }\end{array}$ & $\begin{array}{l}\text { Mulheres grávidas que tiveram } \\
\text { assistência por meio de redes } \\
\text { sociais puderam obter } \\
\text { fornecimento e verificação de } \\
\text { informações e redução de } \\
\text { sentimentos negativos } \\
\text { relacionados ao isolamento. }\end{array}$ \\
\hline 21 & $\begin{array}{l}\text { The need for additional } \\
\text { mental health support for } \\
\text { women in the postpartum } \\
\text { period in the times of } \\
\text { epidemic crisis }\end{array}$ & 2021 & $\begin{array}{l}\text { BMC Pregnancy } \\
\text { and Childbirth }\end{array}$ & $\begin{array}{c}\text { Evidenciar a necessidade de uma } \\
\text { maior atenção à saúde psicológica } \\
\text { da mulher no pós-parto durante a } \\
\text { pandemia de COVID-19. }\end{array}$ & $\begin{array}{l}\text { A pandemia de SARS-CoV-2 } \\
\text { está associada a uma maior } \\
\text { necessidade de cuidados com a } \\
\text { saúde mental da mulher. }\end{array}$ \\
\hline 22 & $\begin{array}{l}\text { Comparison Between In- } \\
\text { Person and Audio-Only } \\
\text { Virtual Prenatal Visits and } \\
\text { Perinatal Outcomes }\end{array}$ & 2021 & $\begin{array}{l}\text { JAMA network } \\
\text { open }\end{array}$ & $\begin{array}{l}\text { Explorar a associação do pré-natal } \\
\text { virtual apenas com áudio com os } \\
\text { resultados perinatais. }\end{array}$ & $\begin{array}{c}\text { Visitas virtuais apenas de áudio } \\
\text { fornecem o cuidado necessário } \\
\text { em uma população sem acesso } \\
\text { imediato aos recursos } \\
\text { tecnológicos de vídeo. }\end{array}$ \\
\hline 23 & $\begin{array}{l}\text { COVID-19 and mental } \\
\text { health during pregnancy: } \\
\text { The importance of } \\
\text { cognitive appraisal and } \\
\text { social support }\end{array}$ & 2021 & $\begin{array}{l}\text { Journal of } \\
\text { Affective } \\
\text { Disorders }\end{array}$ & $\begin{array}{l}\text { Compreender o impacto da COVID- } \\
19 \text { na saúde mental e identificar } \\
\text { fatores de risco e proteção durante a } \\
\text { gravidez. }\end{array}$ & $\begin{array}{l}\text { Isolamento social, problemas } \\
\text { financeiros, dificuldades de } \\
\text { relacionamento e risco de } \\
\text { infecção pela covid-19 são } \\
\text { alguns fatores que interferem na } \\
\text { saúde mental de gestantes } \\
\text { durante a pandemia. }\end{array}$ \\
\hline 24 & $\begin{array}{l}\text { The prevalence and risk } \\
\text { factors of depression in } \\
\text { prenatal and postnatal } \\
\text { women in China with the } \\
\text { outbreak of CoronaVirus } \\
\text { Disease } 2019\end{array}$ & 2021 & $\begin{array}{l}\text { Journal of } \\
\text { Affective } \\
\text { Disorders }\end{array}$ & $\begin{array}{l}\text { Examinar a prevalência de } \\
\text { depressão durante o surto de } \\
\text { COVID-19 em mulheres pré-natais } \\
\text { e pós-natais na China. }\end{array}$ & $\begin{array}{c}\text { Ansiedade, insônia, } \\
\text { movimentação prejudicada e } \\
\text { menor monitoramento do feto } \\
\text { são fatores de risco para } \\
\text { depressão em gestantes durante } \\
\text { a pandemia. }\end{array}$ \\
\hline 25 & $\begin{array}{l}\text { The experiences of } \\
\text { pregnant women during the } \\
\text { COVID-19 pandemic in } \\
\text { Turkey: A qualitative study }\end{array}$ & 2021 & Women and Birth & $\begin{array}{l}\text { Analisar, com base na experiência } \\
\text { individual de mulheres grávidas, } \\
\text { preocupações, problemas e atitudes } \\
\text { de gestantes durante a pandemia. }\end{array}$ & $\begin{array}{c}\text { A pandemia do novo } \\
\text { coronavírus tem potencial } \\
\text { significativo para causar } \\
\text { ansiedade e medo, sendo } \\
\text { necessária atuação da equipe no } \\
\text { acompanhamento da saúde } \\
\text { mental de gestantes. }\end{array}$ \\
\hline 26 & $\begin{array}{c}\text { Pregnant Women's Well- } \\
\text { being and Worry During } \\
\text { the COVID-19 Pandemic: } \\
\text { A Comparative Study }\end{array}$ & 2021 & $\begin{array}{l}\text { BMC Pregnancy } \\
\text { and Childbirth }\end{array}$ & $\begin{array}{c}\text { Investigar preocupações, bem-estar } \\
\text { e outros fatores associados a } \\
\text { gestantes durante a pandemia de } \\
\text { COVID-19. }\end{array}$ & $\begin{array}{l}\text { Baixa renda familiar, situação } \\
\text { profissional e histórico de } \\
\text { aborto são alguns dos preditores } \\
\text { de maior ansiedade nas } \\
\text { mulheres grávidas. }\end{array}$ \\
\hline 27 & $\begin{array}{l}\text { Pregnant under the pressure } \\
\text { of a pandemic: a large- } \\
\text { scale longitudinal survey } \\
\text { before and during the } \\
\text { COVID-19 outbreak }\end{array}$ & 2021 & $\begin{array}{l}\text { European Journal } \\
\text { of Public Health }\end{array}$ & $\begin{array}{l}\text { Estudar as preocupações } \\
\text { relacionadas à saúde de mulheres } \\
\text { grávidas durante a pandemia de } \\
\text { COVID-19. }\end{array}$ & $\begin{array}{l}\text { Mulheres grávidas vivenciam } \\
\text { maiores níveis de ansiedade } \\
\text { durante a pandemia. De forma } \\
\text { semelhante, mas menos intensa, } \\
\text { os parceiros também são } \\
\text { atingidos. }\end{array}$ \\
\hline 28 & $\begin{array}{l}\text { Health anxiety and related } \\
\text { factors among pregnant } \\
\text { women during the COVID- } \\
19 \text { pandemic: a cross- }\end{array}$ & 2021 & BMC Psychiatry & $\begin{array}{l}\text { Avaliar a ansiedade em relação à } \\
\text { saúde de mulheres grávidas } \\
\text { iranianas durante a pandemia de } \\
\text { COVID-19. }\end{array}$ & $\begin{array}{l}\text { As mulheres no terceiro } \\
\text { trimestre apresentaram escores } \\
\text { de ansiedade com a saúde mais } \\
\text { elevados do que as do primeiro }\end{array}$ \\
\hline
\end{tabular}




\begin{tabular}{|c|c|c|c|c|c|}
\hline & sectional study from Iran & & & & e as do segundo trimestre. \\
\hline 29 & $\begin{array}{l}\text { Women's depressive } \\
\text { symptoms during the } \\
\text { covid-19 pandemic: The } \\
\text { role of pregnancy }\end{array}$ & 2021 & $\begin{array}{l}\text { International } \\
\text { Journal of } \\
\text { Environmental } \\
\text { Research and } \\
\text { Public Health }\end{array}$ & $\begin{array}{l}\text { Determinar o risco de sintomas } \\
\text { depressivos em grávidas e mulheres } \\
\text { não grávidas durante a pandemia de } \\
\text { COVID-19. }\end{array}$ & $\begin{array}{c}\text { Alguns fatores de proteção para } \\
\text { sintomas depressivos na } \\
\text { gravidez são o maior apoio do } \\
\text { parceiro, comportamentos } \\
\text { saudáveis e avaliação positiva } \\
\text { da gravidez. }\end{array}$ \\
\hline
\end{tabular}

Fonte: Silva et al. (2021).

\section{Modificações na assistência perinatal, parto e aleitamento materno durante a pandemia de covid-19}

O pré-natal compreende consultas presenciais, aconselhamento e orientações sobre aleitamento materno, nutrição e atividades em grupos, além de ser importante para a redução da mortalidade materna, neonatal e nascimentos prematuros (Duryea et al. 2021). Com o início da pandemia, as consultas de pré-natal foram adaptadas diante da necessidade de manter um acompanhamento seguro, seguindo-se com a avaliação de riscos e diagnóstico de patologias de rotina, com objetivo de resguardar a saúde materna e fetal (Shatilla et al. 2020). As consultas presenciais diminuíram, a telemedicina e grupos virtuais se destacaram como alternativas para garantir e manter a qualidade e humanização do atendimento às gestantes, além de reduzir a exposição ao SARS-CoV-2 (Naurin et al. 2021).

Durante a pandemia, as ações de promoção em saúde, medidas de controle e prevenção contra o novo coronavírus foram importantes para a conscientização das grávidas e puérperas na adoção de medidas de prevenção, diminuindo a possibilidade do adoecimento pela covid-19 e de complicações associadas a um período caracterizado por intensas alterações fisiológicas. Shatilla et al. (2020) considera o enfermeiro como um ator crucial na disseminação de informações seguras e conscientização durante as consultas de pré-natal, por meio de metodologias leves, como cartazes e ilustrações, de uma linguagem simples e clara, embora com frequências reduzidas, as consultas possuíam impactos satisfatórios na troca de informações e fortalecimento do vínculo desse grupo com os profissionais de saúde.

Atualmente, o atendimento de gestantes que apresentam síndrome gripal leve é baseado no manejo clínico, isolamento, orientações e acompanhamento por telefone e entre os fármacos prescritos, como o fosfato de oseltamivir que é recomendado na fase inicial do tratamento (Trapani Júnior et al. 2020). De forma semelhante ao estudo realizado por Reforma et al. (2020) as pacientes grávidas com sintomas da covid-19 passavam pelo processo de triagem, composto por enfermeiros e obstetras, primeiro, por meio de atendimento telefônico. Assim, as pacientes eram avaliadas, direcionadas e orientadas de acordo com os sintomas, sendo que os cuidados de suporte e o isolamento dentro do domicílio constituem a base do tratamento para aproximadamente $80 \%$ dos casos de covid-19. De acordo com o Ministério da Saúde (2020), na classificação em quadros moderados e graves, prioriza-se o bem-estar materno e o devido suporte clínico e recomenda-se a realização de cardiotocografia e ultrassonografia para gestações superiores a 24 semanas. Durante o trabalho de parto, não se deve alterar a via de parto antes de uma avaliação da vitalidade fetal, idade gestacional e condições maternas como a ventilação, devendo priorizar outras tentativas clínicas, antes do parto cesáreo, visto que há possibilidade de piora das condições materna, uma vez que em gestantes com boas condições clínicas o parto vaginal é seguro. O risco de transmissão vertical pode ser provável; no entanto, estudos não demonstram a presença do vírus no líquido amniótico e no leite materno (Trapani Júnior et al. 2020).

A alta hospitalar segue o protocolo da instituição, mas visando o menor tempo de permanência hospitalar, de acordo com a saúde da puérpera e do recém-nascido. Quanto ao acompanhamento puerperal, as orientações sobre lactação e contracepção podem ser virtuais, além de outras consultas de rotina (Ministério da Saúde, 2020). De acordo com ChrzanDętkoś et al. (2021), diante de uma série de restrições hospitalares, o isolamento social associados ao sentimento de insegurança e medo de contaminação podem intensificar problemas psicossociais maternos durante o puerpério, sendo necessário acompanhamento psicológico. 
Quanto à amamentação, há uma série de divergências, porém de acordo com a Organização Mundial de Saúde (2020), mesmo que a mãe esteja infectada pelo SARS-CoV-2, deve amamentar o bebê se estiver em condições clínicas, adotando medidas de higienização das mãos e se possível utilizar máscara. Em casos mais graves, poderá ser feita a extração do leite materno (Trapani Júnior et al. 2020). A amamentação proporciona benefícios nutricionais e imunológicos, além da criação de vínculos entre a mãe e o recém-nascido, esse e outros benefícios da amamentação superam os riscos de acordo com os achados de Cabral, Berezin, Nascimento, e Magalhães (2020).

$\mathrm{Na}$ tentativa de garantir informações seguras, compartilhamento de experiências e buscando criar um ambiente de apoio, o estudo de Chatwin et al. (2021) avaliou o pré-natal por meio de plataformas de mídia social, e as informações compartilhadas em grupos mediados por profissionais de saúde ajudaram na adaptação da rotina, busca de informações e melhora dos cuidados pré-natais. Esse tipo de grupo é importante como rede de apoio e fonte de informações em períodos epidêmicos e de isolamento social. No estudo desenvolvido por Duryea et al. (2021) com o intuito de associar o acompanhamento pré-natal virtual apenas pro áudio com os resultados perinatais, comparando 12.607 mulheres que deram à luz entre $1^{\circ}$ de maio e 31 de outubro de 2019, com aquelas que deram à luz entre $1^{\circ}$ de maio e 31 de outubro de 2020, quando as consultas pré-natais virtuais haviam sido adotadas. $\mathrm{O}$ estudo concluiu que o acompanhamento virtual se mostrou satisfatório para aconselhamento nutricional e outras atividades auxiliares e sem alterações significativas quanto às taxas de diabetes e hipertensão gestacional ou parto prematuro.

Entre muitos desafios à telemedicina encontram-se as condições econômicas, o acesso à Internet, aos dispositivos telefônicos e a dificuldade de estabelecer uma relação pessoal, baseada na confiança, na qual a gestante possa se sentir valorizada e ouvida durante a consulta Ministério da Saúde (2020). Essas dificuldades transpassam tempos de pandemias, porém tornaram-se mais evidentes. Entre as potencialidades da telemedicina, encontram-se a possibilidade de maior da adesão da população com acesso à Internet e dispositivos às atividades relacionadas tanto ao pré-natal quanto ao acompanhamento no puerpério devido à maior facilidade e agilidade no acesso às informações (Reforma et al. 2020).

\section{Isolamento social como fator estressor em gestantes e puérperas}

Durante a pandemia da covid-19 muitas medidas sanitárias foram tomadas ao redor do mundo como tentativa de conter o coronavírus e evitar a contaminação. Ficar em casa e sair apenas para atividades essenciais, manter distanciamento e isolamento social foram maneiras de restringir o contato entre pessoas para evitar que o coronavírus se espalhasse. Mulheres grávidas também estiveram sob as citadas medidas, e por isso experimentaram uma diferente forma de pré-natal, cuidados de saúde e preocupações (Naurin et al., 2020).

Alguns estudos analisados constataram que gestantes estão vivenciando altos níveis de ansiedade e sintomas depressivos durante a pandemia da covid-19. Khoury, Atkinson, Bennett, Jack e Gonzalez (2021) analisaram 303 pacientes grávidas, das quais $57.1 \%$ obtiveram significantes níveis de depressão na escala usada no estudo, divergindo dos tempos de pré-covid, no qual não se tinham níveis de depressão tão altos. As participantes também demonstraram maiores níveis de ansiedade comparados às participantes no período anterior à pandemia e o estudo indicou, ainda, que longos períodos de quarentena estão relacionados a um sofrimento psicológico mais grave. Sahina e Kabakcib (2020) constataram que dúvidas e incertezas sobre os riscos à saúde provocados pela covid-19 no período da pandemia foram fatores que elevaram o medo e a ansiedade entre as entrevistadas.

Mulheres grávidas têm vivenciado estresse psicológico devido aos efeitos secundários da COVID-19 ao mudar seu cotidiano e relações sociais. Fatores como risco de contaminação, isolamento social, dificuldades de relacionamentos e dificuldades financeiras são contribuintes importantes para o desenvolvimento de depressão e ansiedade (Sahina \& Kabakcib, 2020). No entanto, no estudo realizado por Khoury, Atkinson, Bennett, Jack e Gonzalez (2021) com 303 mulheres, embora 
uma parte da amostra das participantes também tenha relatado insônia clinicamente elevada, as taxas de problemas de sono não diferiram das amostras de gravidez pré-covid.

Mulheres com transtornos de ansiedade durante a gravidez podem apresentar sintomas como preocupação, estresse, dificuldade em manter a calma, distúrbios do sono e pensamentos negativos que podem impedir um bom sono (Saadati et al., 2021). Nanjundaswamy et al. (2020) evidenciou em seu estudo realizado na Índia, que o isolamento social e outros quesitos, como mensagens em redes sociais, medo de contrair a infecção, familiares que não seguem os protocolos sanitários e a perda dos rituais relacionados ao parto são as causas mais frequentes de ansiedade e estresse que as pacientes relataram aos obstetras.

O medo do desconhecido, a interrupção do atendimento pré-natal de rotina e a interrupção da vida social por causa da quarentena causaram ansiedade em mulheres grávidas durante a pandemia de covid-19 (Saadati et al., 2021). Em outro estudo, 56.2\% das gestantes de um total de 331 demonstraram preocupação com o fato de ficarem isoladas caso contraíssem o vírus (Zheng et al., 2020). No estudo realizado por Sahina e Kabakcib (2020), as pacientes relataram que atividades como caminhadas que eram importantes para a manutenção da saúde deixaram de ser realizadas devido às medidas restritivas. As participantes também reportaram que houve uma deterioração na expectativa dos cuidados pré-natais, pois não poderiam se dirigir às consultas devido ao medo e isso acabou mudando a forma de realizar o pré-natal.

Além da preocupação com a infecção pelo coronavírus em hospitais, as grávidas também enfrentam dificuldades para se dirigir aos centros de saúde e fazerem os exames devido ao lockdown imposto pelas autoridades. O afastamento social também levou a maiores problemas financeiros e laborais dos casais, e tais questões foram reveladas aos obstetras de forma considerável como um motivo de angústia (Nanjundaswamy et al., 2020). Paralelamente, sintomas mais elevados de depressão e ansiedade foram associados a maior preocupação com as ameaças da covid-19 à vida da mãe e do bebê, bem como preocupações sobre não receber os cuidados pré-natais necessários, tensão no relacionamento e isolamento social devido a pandemia (Lebel et al., 2020).

No estudo realizado por Saadati et al. (2021) com 300 gestantes, 73,6\% das mulheres relataram que a pandemia de covid-19 aumentou sua ansiedade. Uma possível explicação para isso seria a falta de acesso das mulheres a seus provedores de saúde no momento do parto, ou elas poderiam estar relutantes em ir a instalações de saúde ou hospitais, uma vez que consideram esses lugares como sendo ambientes inseguros durante a pandemia de covid-19. Dessa forma, muitas não procuraram atendimento médico para evitar o risco de exposição (Almeida, Shrestha, Stojanac \& Miller, 2020).

As restrições impostas pela pandemia não só provocaram mudanças na rotina das gestantes e puérperas como também alteraram a convivência com outras pessoas e até ampliaram a exposição à situação de violência familiar. Um dos grupos mais vulneráveis à pandemia covid-19 são as mulheres grávidas. Elas não podem escolher abster-se de cuidados; elas e seus filhos correm o risco de complicações graves relacionadas ao vírus; e perdem conforto e apoio quando as clínicas proíbem seus parceiros e as restrições sociais exigem isolamento de amigos e parentes (Naurin et al., 2020).

No estudo de Almeida et al. (2020), o isolamento social se correlacionou fortemente com a probabilidade de depressão ou ansiedade clinicamente significativa. A impossibilidade das participantes de se encontrarem com seus familiares devido ao distanciamento social resultaram em solidão e redução das interações sociais, fazendo com que as gestantes vivessem um processo difícil (Sahina \& Kabakcib, 2020). As preocupações das gestantes podem estar relacionadas ao fato de não terem acesso aos parentes se necessário, e muitas podem ter preocupações com a falta de apoio familiar e social devido às medidas de distanciamento (Saadati et al., 2021).

Devido às diretrizes de isolamento em vigor em muitos lugares, as vítimas de violência praticada pelo parceiro íntimo são privadas da opção de ficar com um amigo ou membro da família ou ir para um abrigo para violência doméstica. Em todo o mundo houve aumentos nas chamadas para linhas de suporte de emergência durante os bloqueios. As ligações para os Serviços de Apoio a Mulheres Maltratadas de Vancouver no Canadá triplicaram. Na França, houve um aumento de 30\% nessas ligações, 
e no Brasil o número é ainda maior (Almeida, Shrestha, Stojanac \& Miller, 2020).

Acerca dos distúrbios psíquicos no pós parto, foi descrito por Mortazavi, Mehrabadi, e KiaeeTabar (2021) que eventos estressantes durante o período de gravidez - como medo, preocupação e ansiedade - podem levar a problemas na relação mãefilho e depressão pós parto. Este estudo revela uma preocupação atual em relação à saúde mental da puérpera devido ao constante receio de uma infecção pelo vírus da covid-19, o isolamento social dessas mulheres e a dificuldade nas relações familiares (Khoury, Atkinson, Bennett, Jack, \& Gonzalez, 2021).

Dentre os artigos analisados para a pesquisa, Lebel et al. (2020) sugerem uma relação direta entre a pandemia pelo SARS-CoV-2 e sérios desafios psicológicos, potenciais de gerar impactos como depressão pós parto em puérperas. Alguns analisaram, ainda, fatores de risco para depressão pós parto no período da pandemia. A maioria das pesquisas estudaram especificamente fatores psicológicos e atitudes do período perinatal e puerpério imediato.

O estudo Lebel et al. (2020) identificou que ansiedade pré natal elevada e sintomas de depressão aumentam o risco de depressão pós parto. Ademais, Almeida, Shrestha, Stojanac, e Miller (2020) concluíram que a diminuição de apoio social - da família, dos amigos e do ambiente de trabalho - está associada com a enfermidade. Enquanto, Li, Huo, Wang, Qi, Wang, Zhou, Zhou, e Zhang (2021) relatam que a diminuição da monitorização do bebê - seja por ultrassom/exame físico - está associada com maior risco para depressão.

Os autores Chrzan-Dętkoś, Walczak-Kozłowska, e Lipowska (2021) apontaram como fator protetor o aleitamento materno até o $3^{\circ}$ mês. Além disso, os resultados desta pesquisa sobre os fatores de risco corroboram com os acima citados e reafirmam como fator de risco o histórico de ansiedade e depressão durante a gravidez. Contudo, trazem como informação adicional que mulheres com este histórico de sintomas depressivos e que param de amamentar cedo têm o risco multiplicado.

\section{Conclusão}

A pesquisa realizada ressalta os impactos negativos da covid-19 na saúde mental materna, na assistência pré-natal e no puerpério. Observando-se que, somada a todas as mudanças e adaptações já esperadas na gravidez, as gestantes lidam com os efeitos secundários da covid-19 que geram restrição de consultas, exames e cuidados pré-natais, diminuição do apoio familiar, preocupações socioeconômicas, medo de transmissão da doença e de suas possíveis complicações. Desta forma, evidencia-se a relação direta entre a pandemia pelo SARS-CoV-2 e os sérios desafios psicológicos, que geram potencial aumento na incidência de ansiedade, depressão entre as gestantes, bem como, depressão pós parto em puérperas. Assim, é significativo o incentivo a capacitação de profissionais multidisciplinares para auxiliar as gestantes no manejo dos fatores estressantes enfrentados.

Dito isto, é importante que mais pesquisas visando avaliar as repercussões da covid-19 à saúde mental de gestantes e puérperas sejam realizadas a partir do ponto de vista de uma equipe multiprofissional, para que possam contribuir no desenvolvimento de intervenções que compreendem aspectos biopsicossociais específicos a esses pacientes.

\section{Referências}

Almeida, M., Shrestha, A. D., Stojanac, D., \& Miller, L. J. (2020). The impact of the COVID-19 pandemic on women's mental health. Archives of women's mental health, 1-8.

An, R., Chen, X., Wu, Y., Liu, J., Deng, C., Liu, Y., \& Guo, H. (2021). A survey of postpartum depression and health care needs among Chinese postpartum women during the pandemic of COVID-19. Archives of Psychiatric Nursing, 35(2), 172-177. https://doi.org/10.1016/j.apnu.2021.02.001

Bandhu Kalanidhi, K., Ranjan, P., Sarkar, S., Kaur, T., Dutt Upadhyay, A., Singh, A., Sahu, A., Khan, M., Vijay Prasad, B., Baitha, U., \& Kumar, A. (2021). Development and validation of a questionnaire to assess socio-behavioural impact of COVID-19 on the general population. Diabetes and Metabolic Syndrome: Clinical Research and Reviews, 15(2), 601-603. https://doi.org/10.1016/j.dsx.2021.02.019

Brown, A. \& Shenker, N. (2021). Experiences of breastfeeding during COVID-19: Lessons for future practical and emotional support. Maternal and Child Nutrition, 17(1), 1-15. https://doi.org/10.1111/mcn.13088 
Cabral, S. A. A. de O., Berezin, E. N., Nascimento, M. B. G., \& Magalhães, S. S. (2020). O Que Esperar no Tocante à Saúde Materno-Infantil no PósPandemia de Covid-19? / What to Expect About Maternal and Child Health in the Post-Pandemic of covid-19? ID on Line REVISTA de PSICOLOGIA, 14(51). https://doi.org/10.14295/idonline.v14i51.2718

Chatwin, J., Butler, D., Jones, J., James, L., Choucri, L., \& McCarthy, R. (2021). Experiences of pregnant mothers using a social media based antenatal support service during the COVID-19 lockdown in the UK: Findings from a user survey. BMJ Open, 11(1), 1-7. https://doi.org/10.1136/bmjopen-2020040649

Chrzan-Dętkoś, M., Walczak-Kozłowska, T., \& Lipowska, M. (2021). The need for additional mental health support for women in the postpartum period in the times of epidemic crisis. BMC Pregnancy and Childbirth, 21(1), 1-9. https://doi.org/10.1186/s12884-021-03544-8

Dib, S., Rougeaux, E., Vázquez-Vázquez, A., Wells, J. C. K., \& Fewtrell, M. (2020). Maternal mental health and coping during the COVID-19 lockdown in the UK: Data from the COVID-19 New Mum Study. International Journal of Gynecology and Obstetrics, 151(3), 407-414. https://doi.org/10.1002/ijgo.13397

Duryea, E. L., Adhikari, E. H., Ambia, A., Spong, C., Mcintire, D., Nelson, D. B., \& Health, P. (2021). Comparison Between In-Person and Audio-Only Virtual Prenatal Visits and Perinatal Outcomes. JAMA network open. 4(4).

Hocaoglu M., Ayaz R, Gunay T, Akin E, Turgut A, \& Karateke A. (2020). Anxiety and post-traumatic stress disorder symptoms in pregnant women during the covid-19 pandemic's delay phase. Psychiatria Danubina [revista en Internet] 2020 [acceso 17 de febrero de 2021]; 32(3-4): 521-526. 32(3), 521-526. http://www.psychiatria-danubina.com/UserDocsImages/pdf/dnb_vol32_no3-4/dnb_vol32_no3-4_521.pdf

Javiera Fuenzalida, C., Caterina Solari, G., Marcelo Farías, J., Rocío Naveas, P., \& José Andrés Poblete, L. (2020). Evaluation of a remote control model for pregnant and post-partum patients with SARS-CoV-2infection. Revista Chilena de Obstetricia y Ginecologia, 85, S35-S49. https://doi.org/10.4067/S071775262020000700007

Khoury, J. E., Atkinson, L., Bennett, T., Jack, S. M., \& Gonzalez, A. (2021). COVID-19 and mental health during pregnancy: The importance of cognitive appraisal and social support. Journal of Affective Disorders, 282(December 2020), 1161-1169. https://doi.org/10.1016/j.jad.2021.01.027

Lebel, C., MacKinnon, A., Bagshawe, M., Tomfohr-Madsen, L., \& Giesbrecht, G. (2020). Elevated depression and anxiety symptoms among pregnant individuals during the COVID-19 pandemic. Journal of Affective Disorders, 277(July), 5-13. https://doi.org/10.1016/j.jad.2020.07.126

Li, C., Huo, L., Wang, R., Qi, L., Wang, W., Zhou, X., Zhou, Y., \& Zhang, X. (2021). The prevalence and risk factors of depression in prenatal and postnatal women in China with the outbreak of CoronaVirus Disease 2019. Journal of Affective Disorders, 282(January), 1203-1209. https://doi.org/10.1016/j.jad.2021.01.019

Ministério da Saúde, Brasil. (2020). Manual de Recomendações para a Assistência à Gestante e Puérpera frente à Pandemia de Covid-19 (recurso eletrônico ed.). Secretaria de Atenção Primária à Saúde. Departamento de Ações Programáticas e Estratégicas. http://189.28.128.100/dab/docs/portaldab/documentos/corona/manual_recomendacoes_gestantes_covid19.pdf

Mizrak Sahin, B., \& Kabakci, E. N. (2021). The experiences of pregnant women during the COVID-19 pandemic in Turkey: A qualitative study. Women and Birth, 34(2), 162-169. https://doi.org/10.1016/j.wombi.2020.09.022

Mortazavi, F., Mehrabad, M., \& KiaeeTabar, R. (2021). Pregnant Women 's Well-being and Worry During the COVID-19 Pandemic : A Comparative Study. BMC Pregnancy and CHildbirth, 4(21), 1-22.

Nanjundaswamy, M. H., Shiva, L., Desai, G., Ganjekar, S., Kishore, T., Ram, U., Satyanarayana, V., Thippeswamy, H., \& Chandra, P. S. (2020). COVID-19related anxiety and concerns expressed by pregnant and postpartum women - a survey among obstetricians. Archives of Women's Mental Health, 23(6), 787790. https://doi.org/10.1007/s00737-020-01060-w.

Naurin, E., Markstedt, E., Stolle, D., Enström, D., Wallin, A., Andreasson, I., Attebo, B., Eriksson, O., Martinsson, K., Elden, H., Linden, K., \& Sengpiel, V. (2021). Pregnant under the pressure of a pandemic: a large-scale longitudinal survey before and during the COVID-19 outbreak. European Journal of Public Health, 31(1), 7-13. https://doi.org/10.1093/eurpub/ckaa223

Organização Mundial da Saúde (2020). OMS- perguntas e respostas sobre COVID-19, gravidez, parto e amamentação. UNA-SUS. Retrieved July $27,2021$. https://www.unasus.gov.br/noticia/oms-perguntas-e-respostas-sobre-covid-19-gravidez-parto-e-amamentacao

Pinos, Morales, VC, Tupiza, SM, Terán, EC e de Aguinaga, RR (2020). Amamentação durante a Pandemia COVID-19: Revisão Narrativa. Ecuadorian Journal of Pediatrics , 21 (1)

Preis, H., Mahaffey, B., \& Lobel, M. (2020). Psychometric properties of the Pandemic-Related Pregnancy Stress Scale (PREPS). Journal of Psychosomatic Obstetrics and Gynecology, 41(3), 191-197. https://doi.org/10.1080/0167482X.2020.1801625

Reforma, L. G., Mph, C. D., Collier, A. Y., Mph, B. J. W., Shainker, S. A., Ms, D. O., Golen, T. H., Herlihy, M., Np, A. L., \& Mph, C. A. Z. (2020). A multidisciplinary telemedicine model for management. The American Journal of Obstetrics \& Gynecology MFM, $2019(\mathrm{January}), 100180$. https://doi.org/10.1016/j.ajogmf.2020.100180

Saadati, N., Afshari, P., Boostani, H., Beheshtinasab, M., Abedi, P., \& Maraghi, E. (2021). Health anxiety and related factors among pregnant women during the COVID-19 pandemic: a cross-sectional study from Iran. BMC Psychiatry, 21(1), 1-7. https://doi.org/10.1186/s12888-021-03092-7

Salehi, L., Rahimzadeh, M., Molaei, E., Zaheri, H., \& Esmaelzadeh-Saeieh, S. (2020). The relationship among fear and anxiety of COVID-19, pregnancy experience, and mental health disorder in pregnant women: A structural equation model. Brain and Behavior, 10(11), 1-8. https://doi.org/10.1002/brb3.1835

Shatilla Misquita, M., Gomes da Silva, P., De Abreu Braz, G., De Almeida Gomes Sousa, A. B., Fontele Catunda Melo, D., \& Nellie de Paula Melo, F. (2020). Atendimento de gestantes na atenção primária a saúde pela enfermagem durante a pandemia do SARS-COV-2. Nursing (São Paulo), 23(269), $4723-4730$. https://doi.org/10.36489/nursing.2020v23i269p4723-4730 
Research, Society and Development, v. 10, n. 10, e484101019186, 2021

(CC BY 4.0) | ISSN 2525-3409 | DOI: http://dx.doi.org/10.33448/rsd-v10i10.19186

Sinaci, S., Ozden Tokalioglu, E., Ocal, D., Atalay, A., Yilmaz, G., Keskin, H. L., Erdinc, S. O., Sahin, D., \& Moraloglu Tekin, O. (2020). Does having a highrisk pregnancy influence anxiety level during the COVID-19 pandemic? European Journal of Obstetrics and Gynecology and Reproductive Biology, 255, 190-196. https://doi.org/10.1016/j.ejogrb.2020.10.055

Taubman-Ben-Ari, O., Chasson, M., Abu Sharkia, S., \& Weiss, E. (2020). Distress and anxiety associated with COVID-19 among Jewish and Arab pregnant women in Israel. Journal of Reproductive and Infant Psychology, 38(3), 340-348. https://doi.org/10.1080/02646838.2020.1786037

Trapani Júnior, A., Vanhoni, L. R., Silveira, S. K., \& Marcolin, A. C. (2020). Protocolo de atenção ao parto, puerpério e aborto durante a pandemia COVID19. Revista Brasileira de Ginecologia e Obstetricia, 42(6), 349-355. https://doi.org/10.1055/s-0040-1713587

Yirmiya, K., Yakirevich-Amir, N., Preis, H., Lotan, A., Atzil, S., \& Reuveni, I. (2021). Women's depressive symptoms during the covid-19 pandemic: The role of pregnancy. International Journal of Environmental Research and Public Health, 18(8). https://doi.org/10.3390/ijerph18084298

Zhao, S. Z., Wong, J. Y. H., Wu, Y., Choi, E. P. H., Wang, M. P., \& Lam, T. H. (2020). Social distancing compliance under covid-19 pandemic and mental health impacts: A population-based study. International Journal of Environmental Research and Public Health, 17(18), 1-11. https://doi.org/10.3390/ijerph17186692

Zheng, Q. X., Jiang, X. M., Lin, Y., Liu, G. H., Lin, Y. P., Kang, Y. L., \& Liu, X. W. (2020). The influence of psychological response and security sense on pregnancy stress during the outbreak of coronavirus disease 2019: A mediating model. Journal of Clinical Nursing, 29(21-22), 4248-4257. https://doi.org/10.1111/jocn.15460 